\title{
Judith Butler en Argentina. Recepción y polémicas en torno a la teoría de la performatividad del género
}

\begin{abstract}
Resumen: En idioma español, las ideas y conceptualizaciones de Judith Butler han circulado por más de dos décadas. Su recepción no fue uniforme ya que, en cada país de habla hispana, estuvo condicionada por el interés y las resistencias que generaron sus arriesgadas tesis en los feminismos y en los Estudios de Género, por los anacronismos propios de la traducción y por las dinámicas propias de la academia y de la industria editorial. En Argentina, las primeras traducciones de textos de Butler comienzan a publicarse y circular en la década del noventa en revistas que no estaban totalmente dedicadas a la teoría o al quehacer académico, pero tampoco al activismo desprovisto de conceptos y modos de pensar académicos. Este artículo explora las distintas vías de recepción, lectura y utilización de los conceptos de la teoría de la performatividad del género en Argentina, así como también las polémicas que se generaron en torno a ella, focalizando sobre las tensiones y los cruces entre el activismo y la academia, dos campos que propongo pensar como espacios de disputas con fronteras mucho más frágiles y porosas de lo que muchas veces se supone.
\end{abstract}

Palabras clave: Judith Butler; performatividad; recepción; Argentina.

\section{Introducción}

Este trabajo rastrea y analiza las distintas vías de recepción, lectura y utilización de los conceptos de la teoría de la performatividad del género de Judith Butler en Argentina. Para su elaboración, se siguieron las indicaciones de perspectivas teóricas y estudios acerca de la circulación y recepción de ideas que conciben a la lectura y al uso de conceptos en contextos distintos a los de su producción como un proceso complejo, activo y creativo (Roberto SCHWARZ, [1973] 2014; Pierre BOURDIEU, 1999; Horacio TARCUS, 2007,

\section{(c) (i)}

Esta obra está sob licença Creative Commons. 
2016; otros). En línea con estos estudios, este trabajo no se propone dilucidar si es o no correcto utilizar conceptos provenientes de otras latitudes, y tampoco realiza un juicio sobre la fidelidad o infidelidad de tal o cual lectura, lo cual presupondría la atribución de una suerte de objetividad a las ideas y a los textos en su "origen". Se trata, por el contrario, de indagar en los modos en que las ideas de Butler fueron interpretadas y utilizadas para intervenir en discusiones locales donde, como veremos, lo conceptual no estaba desligado de lo político. El trabajo de investigación procedió revisando con minuciosidad materiales publicados, principalmente en revistas y libros. En dichos materiales interesó sobre todo rastrear la difusión, los usos y las polémicas generadas en torno a los textos de Butler entre la década del noventa y comienzos de la primera década de este siglo. El análisis revela tanto el importante rol de las redes feministas de lectoras, editoras, traductoras y autoras, como también las tensiones y los cruces entre dos campos que, a priori, se presentan como estructurados oposicional y dicotómicamente a la vez que estructurantes de una buena parte de los debates feministas y queer contemporáneos: el "activismo" y la "academia". Se propone reflexionar acerca de la recepción de la obra de Butler en Argentina desde una perspectiva que pone en tensión estas dicotomías, al mismo tiempo que advierte la porosidad y fragilidad de sus fronteras en aquella coyuntura histórica. Finalmente, se presenta un esbozo inicial acerca de los usos actuales y los desafíos que plantea la utilización de las conceptualizaciones de Judith Butler en Argentina.

En idioma español, el recorrido de las ideas y conceptualizaciones de Judith Butler tiene ya más de dos décadas. Pero su recepción no fue uniforme ya que, en cada país de habla hispana, estuvo condicionada por el interés y las resistencias que generaron sus arriesgadas tesis en los feminismos y los Estudios de Género, por los anacronismos propios de la traducción y por las dinámicas propias de la industria editorial. La primera edición de El género en disputa. El feminismo y la subversión de la identidad fue publicada en México recién en el año 2001, once años después de la publicación original de Gender Trouble. Feminism and the Subversion of Identity.

En Argentina, hasta la aparición de dicha publicación, el interés en la obra de Judith Butler fue creciendo al compás de la circulación tanto de textos en idioma original como de traducciones que comenzaron a publicarse y a circular en la década del noventa en publicaciones que no estaban totalmente dedicadas a la teoría o al quehacer académico, pero tampoco al activismo desprovisto de conceptos y vínculos con la vida académica. ${ }^{1}$

Feminaria Editora y su revista, Feminaria, fueron usinas clave a través de las cuales los textos de Judith Butler comienzan a ser traducidos y publicados. Ambas formaban parte del mismo proyecto editorial feminista impulsado por mujeres provenientes en gran parte del ámbito universitario y con variada participación en los emergentes Estudios de Género. En 1992, Feminaria Editora publica el libro Feminismo/posmodernismo, que consiste en la selección y traducción de cuatro ensayos que formaban parte de la compilación Feminism/ Postmodernism (Routledge, 1990) realizada por Linda Nicholson. Entre dichos ensayos se encontraba la primera traducción publicada en Argentina de un texto de Judith Butler: "Problemas de los géneros, teoría feminista y discurso psicoanalítico" (trad. Márgara Averbach).

En 1997, Feminaria publica, en su número 19, el artículo "Sujetos de sexo/género/ deseo", una traducción del primer capítulo de Gender Trouble (Routledge, 1990), realizada por Adolfo Campoy Cubillo. El siguiente número, publicado en el mismo año, contiene el texto "Las feministas teorizan lo político". Una traducción realizada por Teresa Azcárate y

' En relación con las revistas feministas donde se publicaron estas primeras traducciones, este trabajo tiene como antecedente fundamental el publicado por Paula Torricella (2013). Véase también, Mabel Bellucci (2015). 
Alicia Ferreira de la introducción del libro Feminist Theorize the Political (Routledge, 1992), escrita por Judith Butler y Joan Scott. Feminaria continúa con su tarea de traducción y difusión en 1999 con la publicación de "La vida psíquica del poder...", introducción del libro The Psychic Life of Power: Theories in Subjection (Stanford University Press, 1997) traducida por Márgara Averbach. La revista Feminaria no editorializa los textos de Butler ni los pone en relación con otros, pero lleva adelante una importante tarea de difusión que animó ciertos debates en curso a nivel local en torno al sujeto político del feminismo y a la relación entre el feminismo y el postestructuralismo (TORRICELLA, 2013).

En 1998 aparece en la revista mexicana Debate feminista un artículo clave de Judith Butler traducido por Marie Lourties, publicado originalmente en 1990, y que circularía con intensidad en Argentina durante los siguientes años: "Actos performativos y constitución del género: un ensayo sobre fenomenología y teoría feminista". También en 1998, pero en Argentina, el número 4 de la revista Mora-del Instituto Interdisciplinario de Estudios de Género de la Universidad de Buenos Aires- publica un dossier titulado "Butler lee a Beauvoir", con traducción, selección y notas de la filósofa María Luisa Femenías. Dicho dossier marca el inicio del intenso y fructífero diálogo de Femenías con la obra de Butler. Allí se incluye la traducción del artículo "Sexo y género en El segundo sexo de Simone de Beauvoir" (BUTLER, [1986] 1998a) (originalmente publicado en la revista Yale French Studies) y también la traducción de algunos fragmentos de dos capítulos de Gender Trouble bajo el título "El primer libro de Judith Butler: Los problemas de género" ([1990] 1998b). Dichas traducciones estaban acompañadas por dos textos que establecían un diálogo crítico con los de Butler: "Butler lee a Beauvoir: Fragmentos de una polémica en torno del "sujeto" (FEMENÍAS, 1998) y "¿Qué es ser mujer? Butler y Beauvoir sobre los fundamentos de la diferencia sexual" (trad. Larissa Zadorojny) (Sara HEINÄMAA, 1998). En Mora, entonces, se establece de manera más clara que en Feminaria un diálogo con los textos de Judith Butler que tuvo la característica de darse en clave filosófica y de centrarse en las lecturas de Simone de Beauvoir (TORRICELLA, 2013).

Otro ámbito temprano de lectura y recepción de la teoría de la performatividad en Argentina fue el psicoanálisis. Mayormente, los textos de Butler se leyeron entre profesionales que se especializaron en cuestiones de género y sexualidad desde una perspectiva crítica de las líneas freudianas y lacanianas ortodoxas. En el año 2000 la Editorial de la Escuela Lacaniana de Psicoanálisis publica un volumen titulado Grafías de Eros. Historia, género e identidades sexuales que contenía, entre otros textos, el artículo de Butler "Imitación e insubordinación de género", publicado originalmente en 1991 (trad. Mariano Serrichio).

Durante la primera década del 2000 , y sobre todo en su segunda mitad, los textos de Judith Butler en español comienzan a circular con mayor fluidez, en parte porque la industria editorial empieza a interesarse en ellos. Su obra nos llega, como sostienen Patricia SoleyBeltrán y Leticia Sabsay (2012) "toda junta" en esa década, cuando sus libros comienzan a traducirse cada vez con mayor rapidez, al punto de que algunos de sus textos más recientes se editan en español y se comercializan en Argentina apenas un año después de su publicación original. También, la expansión de Internet y de los medios digitales de copiado a partir de estos años colaboraron a que los textos, ya sea en inglés o en español, circularan con mayor intensidad y fluidez. La innumerable cantidad de ediciones "pirata" de los textos de Butler en español, la avidez por sus novedades editoriales y por asistir a sus conferencias en las dos oportunidades en que visitó Argentina, así como también la publicación de numerosos trabajos de revisión crítica de sus textos muestran el gran interés que su obra genera dentro y fuera de las instituciones académicas.

En un primer momento de la recepción en Argentina entre la segunda mitad de la década del noventa y la primera mitad de la década del 2000 , los textos de Butler no se 
leyeron solos, sino que comenzaron a estudiarse junto con los de otras feministas postestructuralistas como Donna Haraway, Rosi Braidotti, Joan Scott y Teresa de Lauretis, entre otras. Con sus diferencias, las intervenciones de esas teóricas feministas vinieron a conmocionar un campo de estudios académicos, reflexión teórica y acción política que en Argentina, desde la segunda mitad de la década del 1980 comenzaba a estabilizarse en torno a ciertos conceptos e identidades políticas ("género" y "mujeres", principalmente). ${ }^{2}$ Las críticas "post" se dirigían tanto hacia la fijeza esencialista del sujeto presupuesto por las teorías y las prácticas feministas, como a la rigidez de los marcos binarios que, hasta entonces, habían sustentado las reflexiones sobre el género, el cuerpo y la identidad. Así como la noción de una unidad de las "mujeres" fue puesta en crisis por estas teóricas feministas, también ciertos usos de la categoría "género" comenzaron a ser objeto de críticas, y se apostó a la revisión del estatuto del "sexo" como fundamento corporal último del sujeto político y teórico del feminismo. Los textos de Butler comienzan a ser citados en esos años como parte de esta profunda -y también, ciertamente, incómoda- renovación teórica que, en ciertos sectores de los feminismos académicos y no académicos, fue recibida como un estímulo para repensar las prácticas de investigación y también la praxis política feminista. ${ }^{3}$

En el siguiente apartado mostramos que, incluso, los estudios más apegados a la discusión filosófica incluyeron una valoración de la productividad política de las conceptualizaciones de Butler. Sus textos, lejos de estimular una discusión meramente teórica, provocaron un desafío a los modos establecidos de hacer y de reflexionar acerca de la política en el campo de las izquierdas, los feminismos y los activismos socio-sexuales. Surgieron, por ejemplo, preguntas acerca de qué acciones políticas serían posibles teniendo en cuenta la crítica furibunda a las políticas identitarias, a la representación política y a la noción de sujeto auto-centrado. De modo que esta teoría y sus conceptos no funcionaron en su llegada a Argentina como un discurso de autoridad especulativo y desligado de la práctica política, sino como operadores que provocaron e impulsaron un intenso debate conceptual y político situado.

\section{Lecturas críticas entre la filosofía y la política}

La filósofa María Luisa Femenías fue una de las primeras estudiosas del pensamiento de Judith Butler y continúa siendo una de sus lectoras más agudas y sistemáticas. Femenías publica, como dijimos, artículos pioneros en los que analiza críticamente el diálogo que Judith Butler establece, en sus textos tempranos, con Simone de Beauvoir (FEMENÍAS, 1998; 1999; [2000] 2012). El libro Judith Butler: Introducción a su lectura (FEMENÍAS, 2003) ofrece un análisis filosófico más amplio centrado en la noción de sujeto y en la revisión crítica de la reformulación butleriana de conceptos y nociones fundamentales para las teorías feministas como sexo, mujeres, género, cuerpo y diferencia, entre otras. En dicho libro, Femenías realiza una matizada elaboración crítica de un pensamiento que reconoce en pleno desarrollo y del cual destaca su complejidad y su riqueza. Sostiene, con argumentos basados en una lectura minuciosa, que "Ias posibilidades que Butler ofrece al feminismo real son hasta hoy políticamente limitadas" (FEMENÍAS, 2003, p. 6) y rechaza el desplazamiento de las "mujeres" como sujeto político del feminismo argumentando que

${ }^{2}$ Una pormenorizada revisión histórica del recorrido de los feminismos académicos en Argentina puede encontrarse en FEMENÍAS (2005).

${ }^{3}$ Véanse, entre otros: Nora DOMÍNGUEZ; Marcela CASTRO; July CHÁNETON; Silvana DASZUK y Silvia JUROVIETZKY (1994); CHÁNETON (1998); Teresa AZCÁRATE, María Elena BARTís y Silvia WERTHEIN (1999); Ana AMADO, (2000); Paula HALPERÍN y Omar ACHA (2000). 
"[d]e la proliferación sin más de sexos-géneros no se sigue necesariamente que la dominación y las jerarquías se acaben: por el contrario, pueden adquirir nuevas y complejas formas" (FEMENÍAS, 2003, p. 79).

Su valoración del pensamiento de Butler, en este punto, es profundamente política, a pesar de que sus lecturas críticas y problematizaciones han estado guiadas por un interés teórico y filosófico centrado, en buena parte, "en el problema ontológico, más que político, de la constitución del sujeto" (FEMENÍAS, 2003, p. 5). Es posible observar que, incluso en una vía de recepción que podría caracterizarse como la más propiamente teórica y conceptual, el interés de la lectura se desplaza necesariamente hacia la cuestión de la praxis política y de las posibilidades que tienen esos conceptos de ser operativos para las disputas feministas.

En una línea de lectura también crítica de la recepción y de las implicancias políticas de los usos de los conceptos de Butler en Argentina, encontramos los artículos de Alejandra Ciriza (2004, 2006, 2010), Andrea D’Atri (2003) y Yuderkis Espinosa Miñoso (2004). Consideramos que las enérgicas críticas de estas autoras pueden estructurarse tentativamente en torno a cuatro ejes que serán retomados sobre el final de este texto: 1) la cuestión del uso de teorías provenientes de otras latitudes, 2) la cuestión del estatuto de los cuerpos, 3) la cuestión de la importancia de la explotación capitalista y 4) la cuestión de la relación entre la performatividad butleriana, el activismo trans y los feminismos.

En cuanto al primer punto, en "Notas sobre los límites de la importación teórica. A propósito de Judith Butler", Alejandra Ciriza expresa preocupación por la "hipnosis que Butler produce en el mundo académico" (CIRIZA, 2004, p. 60). Realiza un llamado a tener en cuenta las "marcas de origen de las teorías" (CIRIZA, 2004, p. 59) y a considerar que "Ias actuales condiciones de producción académica favorecen la importación de herramientas teóricas y conceptuales acuñadas en la academia norteamericana sin la suficiente vigilancia epistemológica" (CIRIZA, 2004, p. 58). Debido a ello, considera necesario tener en cuenta las dificultades en la traducción de los contextos políticos y las jerarquías entre quienes teorizan y militan en el norte y en el sur.

En sus artículos, Ciriza ha criticado asimismo lo que ella caracterizó como una "apuesta a la disolución de la materialidad real del cuerpo" (CIRIZA, 2004, p. 58) en la propuesta teórica de Butler. Para argumentar en ese sentido, utiliza herramientas del psicoanálisis en un artículo posterior, y sostiene que Butler propone "la disolución de la distinción entre el orden de lo real y el orden de lo simbólico" (CIRIZA, 2006, p. 35). En cambio, apuesta en su texto por "pensar lo real como la insistencia en lo que la falta, el límite, la carencia constitutiva de tod@sujeto, su mortalidad, la vulnerabilidad de su cuerpo, pero también el goce sin palabras, amenazan los sentidos establecidos" (CIRIZA, 2006, p. 39).

Otro eje de críticas atribuyó a Butler un desinterés por los procesos de exclusión y explotación capitalista. Para Ciriza, la teoría de Butler se detiene ante "lo impronunciable del capitalismo, la brutal materialidad de la dominación sobre los cuerpos" (CIRIZA, 2004, p. 60). En una línea similar, Andrea D'Atri (2003) critica los usos locales de Butler, a los que caracteriza como parte de una "moda" teórica. Dirige sus objeciones a las elaboraciones butlerianas acerca de la democracia radical, y sostiene que no aportan una crítica del "Estado democrático burgués, basado en la explotación capitalista".

Por último, en cuanto a la cuestión de la relación entre la performatividad, el activismo trans y los feminismos, Yuderkis Espinosa Miñoso en su artículo "A una década de la performatividad: de presunciones erróneas y malos entendidos" (2004) define al impacto de El género en disputa como un "quiebre" cuyo efecto, según ella, ha sido el "auge" de la identidad travesti acompañado de la heterosexualización del movimiento feminista y un concomitante borramiento de la identidad lesbiana en el movimiento feminista y queer. 
La obra de Butler ha suscitado en su recorrido transnacional intensas polémicas de las cuales se ha nutrido y, como vimos, Argentina no ha sido la excepción. Además de estas lecturas críticas, es posible rastrear en nuestro contexto local una vía de recepción entusiasta con respecto a los textos y conceptos de Judith Butler que tuvo un carácter fuertemente situado, crítico y comprometido con las luchas políticas que se agitaban en aquel momento histórico.

\section{Usos entusiastas y políticas feministas, queer y trans}

El número 1 1/12 de la revista El rodaballo, publicado en el año 2000, incluyó una sección compuesta de dos artículos y titulada "Feminismo y socialismo: En torno al debate Butler/Fraser", en donde es posible advertir el interés que dicho debate generaba tanto en los feminismos como en sectores de la izquierda no ortodoxa (Claudia BACCl, Laura FERNÁNDEZ CORDERO y Alejandra OBERTI, 2000; Omar ACHA, Pablo BEN, Débora D'ANTONIO, Paula HALPERÍN y Daniela LAGO, 2000). Ambos artículos retoman y comentan críticamente la polémica entre Judith Butler y Nancy Fraser que El rodaballo había publicado en números anteriores (BUTLER, 1999a; FRASER, 2000). En su artículo, Bacci, Fernández Cordero y Oberti destacan el aporte de Butler "para pensar la política feminista sin sujetos preconstituidos". En ese sentido, y llevando hasta las últimas consecuencias la afirmación de que "no hay un locus prefijado desde el cual se vaya a producir la falla del orden hegemónico" (BACCl, FERNÁNDEZ CORDERO y OBERTI, 2000, p. 90), la propuesta de las autoras se dirige a mostrar que no hay un tipo de práctica ni de sujeto sexual que, a priori, pueda llamarse no normativo y tenga una posición preferente para trastocar el orden socio-sexual. Las autoras valoran positivamente la no determinación del sujeto político del cambio social que realiza Butler, pero no describen sus ideas de manera acrítica, sino que le plantean interrogantes. Efectúan una lectura situada que parte de la pregunta " $¿$ [q]ué significa leer a estas autoras en la Argentina del 2000?" y se esfuerzan por poner en juego esas discusiones en cuestiones del presente político local como la sanción de la Ley de Salud Sexual y Reproductiva y la prostitución. De modo que, en ese texto muy temprano, los conceptos y su revisión no están desligados de la crítica y tampoco de una reflexión feminista localizada.

La lectura temprana de Butler en Argentina no estuvo restringida a los feminismos y los Estudios de Género, sino que a su vez estuvo fuertemente relacionada con la emergencia de grupos académicos y de intervención política autodenominados queer que tuvieron una fuerte presencia activista y un trabajo académico interesado en la reflexión sobre las luchas socio-sexuales en curso por aquellos años. Según José Maristany, estos grupos colaboraron "... a diversificar la propuesta ofrecida hasta ese momento por las áreas e institutos de estudios de la mujer y de género en la academia" (MARISTANY, 2013, p. 107). Aunque tomaron su denominación de la Teoría Queer emergente en Europa y Estados Unidos y una de cuyas referentes ineludibles es Judith Butler, sería aventurado decir que se trataba de meros procesos de importación acrítica de teorías metropolitanas. Sus intervenciones establecieron un diálogo y un trabajo de traducción de los conceptos de la teoría queer que se inscribía en una línea genealógica de producción poética, académica y política anterior, vinculada especialmente con el trabajo de Néstor Perlongher (Cecilia PALMEIRO, 2014). Tampoco sería justo decir que se trató de grupos que apoyaban demandas liberales centradas únicamente en la visibilización de diferencias asociadas con la identidad sexual, ya que, por el contrario, se constituyeron como sujetos colectivos de reclamos contra la violencia represiva que se inscribían en la larga historia de resistencias contra los gobiernos dictatoriales en Argentina (Carlos FIGARI, 2014).

Flavio Rapisardi -uno de los principales animadores del Área de Estudios Queer de la Facultad de Filosofía y Letras de la Universidad de Buenos Aires- publicó varios artículos 
tempranos en los cuales el nombre de Judith Butler aparece junto con el de otras feministas y teóricas queer en el marco de reflexiones sobre los Estudios Culturales y sobre las polémicas en torno a la identidad y la diferencia (RAPISARDI, 1997, 1998, 1999). En abierta polémica con "la fobia anti-Butler en el feminismo" y con la "izquierda académica" que "exigía carta de nacionalidad teórica" (RAPISARDI, 2008), la emergente perspectiva queer local se comprometió con la crítica de las identidades fijas sin renunciar al pensamiento sobre las desigualdades atravesadas por la dinámica capitalista y neoliberal. En este sentido, fueron perspectivas que no tuvieron como horizonte último la "visibilidad", la "inclusión" o el "reconocimiento", sino que, de manera más amplia, buscaban impulsar una "transformación social, económica y cultural " que hiciera hincapié en lo que llamaron "el valor crítico de la diferencia” (BELLUCCI y RAPISARDI, 1999). ${ }^{4}$

En aquellos años de profunda crisis política, económica y social pero también de gran activación de luchas sociales, dos conflictos operaron como trasfondo de las polémicas en torno a la recepción de los textos de Judith Butler. Por un lado, la disputa por la participación o no de las travestis en los Encuentros Feministas y en los Encuentros Nacionales de Mujeres. Y, por otro lado, las luchas en torno al Código Contravencional porteño y sus reformas, que tuvo como protagonistas a las travestis organizadas contra la criminalización tanto del trabajo sexual como de su circulación en el espacio público de la ciudad.

En cuanto al primer escenario de conflicto, la conmoción política que se agitaba en el campo de los feminismos por aquellos años giraba en torno a ciertas preguntas: ¿Podían las travestis ser sujetos legítimos del feminismo? ¿Podían llamarse a sí mismas "mujer" o "feminista" quienes no tuvieran un cuerpo sexuado considerado femenino según los criterios biomédicos dominantes? ¿El cuerpo travesti y sus formas de recrear los rasgos culturalmente asociados con la "feminidad" reproducían o contradecían los estereotipos de género? Estas preguntas no se formulaban en abstracto por la lectura de los textos de Judith Butler ni pueden reducirse a los efectos de una "moda" teórica foránea, sino que surgían de la inquietud que provocaban las luchas y la visibilidad del emergente activismo travesti y sus tensas alianzas con algunos sectores de los feminismos y con el activismo queer. Las conceptualizaciones de Butler fueron utilizadas - en esa coyuntura de profunda crisis social y de reactivación de las luchas de las mujeres en Argentina - para fortalecer esas alianzas y también las posiciones del activismo travesti.

Sobre aquella disputa y el encuentro de las travestis con los feminismos antiesencialistas locales, Lohana Berkins sostiene:

...compartiendo (...) con compañeras feministas, algunas de nosotras comenzamos a levantar las banderas de este movimiento e incluso a definirnos también como feministas. No obstante, la mirada de algunas de ellas sobre nosotras sigue situándonos en nuestro origen biológico masculino. De ahí que en el año 1996 se nos prohibiera la entrada a las jornadas feministas que se realizan anualmente. De ahí también que bastante después se nos prohibiera la entrada a uno de los encuentros nacionales feministas, realizado este en la ciudad de Río Ceballos, Córdoba. (BERKINS, [2003] 2008, p. 148-149)

Una reflexión sobre la polémica desatada en torno a la participación de travestis en el Encuentro Feminista de Río Ceballos en el año 2000 puede leerse en el número 24/25 de Feminaria bajo el título "Las desventuras del género" (Liliana AZARAF, Silvia CATALÁ, Liliana DAUNES, Mónica D'UVA, Josefina FERNÁNDEZ y Silvia VICENTE, 2000). Allí, quienes apoyaron el taller sobre feminismo y travestismo citan y utilizan argumentos butlerianos para discutir las limitaciones que supone restringir el feminismo a las mujeres y a una determinada

${ }^{4}$ Sobre la emergencia y las utilizaciones de lo queer en Argentina véanse, entre otros: Silvia Delfino (1999); Fabricio Forastelli (2007, 2010); Delfino y Rapisardi (2010); Palmeiro (2012) y Figari (2014a). 
configuración genital. ${ }^{5}$ En esta coyuntura política, un conjunto de feministas con posturas críticas y antiesencialistas se constituirán en aliadas cercanas del emergente movimiento travesti y de sus referentes, estableciendo grupos de formación, debate y activismo conjunto. ${ }^{6}$ La otra contienda política que se agitaba por aquellos años y que articuló la emergente lucha travesti con intervenciones académicas feministas y queer estuvo relacionada con el escenario posterior a la autonomización de la Ciudad de Buenos Aires (en 1996), cuando se derogaron los Edictos Policiales y se sancionó, en 1998, un nuevo Código Contravencional que fue denominado "Código de Convivencia Urbana". En consonancia con el espíritu garantista y democrático de ese nuevo Código, las identidades trans no fueron criminalizadas a priori como sí sucedía mientras regían los Edictos Policiales. Sin embargo, en las sucesivas modificatorias y reformas del Código, las disputas y los debates se desplazaron hacia la regulación del trabajo sexual callejero, lo cual afectaba especialmente y criminalizaba a travestis y trans, quienes se organizaron para resistir a esos embates.

Esas luchas, que se desarrollaron tanto en el espacio callejero como en los medios de comunicación y en el ámbito legislativo, provocaron otros productivos encuentros entre grupos queer con pertenencia académica y el activismo travesti. Los y las integrantes del Área de Estudios Queer de la Facultad de Filosofía y Letras de la Universidad de Buenos Aires produjeron agudas intervenciones críticas acerca de dichas reformas que abrieron líneas de alianza política, de activismo y de indagación socio-cultural que aún permanecen abiertas. $^{7}$

El encuentro entre las emergentes organizaciones travestis y los grupos de feministas antiesencialistas y queer que tenían inscripciones variadas en el ámbito universitario resultó en un original activismo que articuló -con tensiones- la lucha por derechos y reconocimiento social con una crítica social y cultural en términos amplios en momentos de profunda crisis político-institucional y económica. La lucha por el derecho a la identidad asociada con el género consagrado en 2012 por la Ley de Identidad de Género ( $N^{\circ} 26743$ ) estuvo vinculada, desde sus inicios, con el reclamo de otros derechos, como el derecho a la salud, al trabajo, a la vivienda y el derecho a la asociación.

También de ese encuentro surge una temprana producción de saberes críticos sobre las cuestiones trans, el travestismo, la transexualidad y la transgeneridad, que tuvo como sujetos de enunciación no solo a quienes tenían inscripciones en el ámbito universitario sino también a las/os activistas travestis y trans en diálogo -muchas veces polémico con teorías postestructuralistas para comprender los géneros, las sexualidades, los cuerpos y la política, en las cuales Butler tenía un lugar central.

La compilación Sexualidades migrantes. Género y transgénero, realizada por Diana Maffía y publicada originalmente en 2003 (2008), es la primera dedicada a la discusión acerca de la cuestión intersex, travesti, transexual y transgénero desde una perspectiva social, política, cultural e histórica que tiene como horizonte la lucha por derechos ciudadanos e incluye críticas al feminismo esencialista. Allí, Judith Butler es recurrentemente citada en distintos artículos donde se recupera su crítica a la naturalización del orden binario de los sexos y los géneros. También se retoma su crítica a la fijeza y el carácter excluyente del sujeto del feminismo cuando se reduce a la categoría identitaria "mujeres".

En 2004 se publica la compilación Cuerpos ineludibles (Josefina FERNÁNDEZ, Mónica D'UVA y Paula VITURRO, 2004), donde es posible encontrar un señero artículo de la activista

\footnotetext{
${ }^{5}$ Sobre la participación de las travestis en los Encuentros Nacionales de Mujeres, véase Amanda Alma y Paula Lorenzo (2009).

"Véase, "Entrevista con Lohana Berkins" (BERKINS y Daniel JONES, 2008).

${ }^{7}$ Véanse, entre otros: Delfino y Guadalupe Salomón (2003); Rapisardi (2005); ÁREA QUEER (2006); llona Aczel y Juan Péchin (2006); Salomón $(2011,2012)$.
} 
travesti Lohana Berkins acerca del activismo travesti y también el ya mencionado artículo crítico acerca de la performatividad butleriana de Yuderkis Espinosa Miñoso. En el mismo año se publica Cuerpos desobedientes. Travestismo e identidad de género, de Josefina Fernández (2004). Se trata del primer estudio sobre la identidad travesti, realizado desde una perspectiva antropológica y surgido del encuentro de la autora-autodefinida feminista- con el movimiento travesti. Allí, y también en un artículo posterior (FERNÁNDEZ, 2007), la autora se desplaza de los marcos interpretativos antropológicos y médicos más usuales en aquel momento, para tomar como herramientas las conceptualizaciones de Judith Butler en conjunción con otras que le permiten producir una investigación situada y en claro diálogo con las demandas de la comunidad travesti.

La producción de conocimientos sobre la identidad travesti continúa con la publicación, en 2005, de La gesta del nombre propio. Informe sobre la situación de la comunidad travesti en la Argentina coordinado por la activista travesti Lohana Berkins junto con Josefina Fernández. Se trata de los resultados de una investigación organizada por la Asociación de Lucha por la Identidad Travesti y Transexual (ALITT) donde se busca dar cuenta de las condiciones de vida de las travestis, transexuales y transgéneros para mostrar la violencia y la exclusión del colectivo de los más básicos derechos ciudadanos. Esa producción de conocimiento, impulsada por el activismo, en alianza con personas inscriptas en espacios académicos y orientada a la lucha por derechos, continúa con Cumbia, copeteo y lágrimas. Informe nacional sobre la situación de las travestis, transexuales y transgéneros (BERKINS, 2007).

A partir de estos años, el trabajo intelectual y político de activistas trans e intersex se hace visible en numerosas publicaciones dirigidas a impulsar la producción de conocimiento crítico como herramienta para la transformación social. Entre otros, cabe mencionar los trabajos de Lohana Berkins ([2003] 2008, 2004, 2010, 2013, otros), Marlene Wayar quien es, además, directora de El Teje: Primer Periódico Trans Latinoamericano (2005, 2007, 2012, 2013, otros), Mauro Cabral ([2003] 2008, 2009, 201 1, 2012, otros; CABRAL y VITURRO, 2006) y Blas Radi $(2013,2015$, otros).

Este amplio y diverso conjunto de saberes críticos tiene la peculiaridad de haber sido construidos en una buena parte por referentes travestis, trans e intersex con el objetivo de dar a conocer las problemáticas que los/as afectan, fortalecer sus demandas políticas, y también emprender una crítica del sistema cisexista que jerarquiza y distribuye privilegios inequitativamente entre personas trans y no trans. En este último grupo de trabajos publicados ya entrada la década del 2000 es posible observar que no tienen un lugar destacado los textos canónicos de la teoría queer, del feminismo postestructuralista, ni de Judith Butler. Podría decirse que se observa en ellos una relación crítica con este conjunto de textos, una progresiva autonomización respecto de sus conceptos para reflexionar acerca de las cuestiones trans y un pasaje hacia valiosas formas de reflexión y teorización trans latinoamericana que establece conexiones con emergentes saberes y teorizaciones trans de otras latitudes.

\section{Cómo se usan los conceptos de la teoría de la performatividad en Argentina: coordenadas iniciales}

En el polémico campo interdisciplinario de los Estudios de Género y Sexualidades, Feministas y Queer, numerosos trabajos se han ocupado, en la última década, de revisar distintos aspectos conceptuales del pensamiento de Judith Butler. En el campo de la filosofía e impulsada en gran medida por el extenso y sostenido trabajo de María Luisa Femenías en el Centro Interdisciplinario de Investigaciones en Género de la Universidad de La Plata su 
recepción ha sido intensa. En años más recientes, se destacan trabajos con el interés puesto en el estudio de la cuestión central del deseo (Rolando CASALE, 2006; CASALE y Cecilia CHIACCHIO, 2009) y de la desustantivización del sujeto en la obra de Butler (FEMENÍAS, 2013). Asimismo, un renovado y creciente interés filosófico puede advertirse en las numerosas tesis de licenciatura y de doctorado en filosofía que en los últimos años estuvieron dedicadas a estudiar y problematizar filosóficamente distintos aspectos de la obra de Judith Butler (Mariela SOLANA, 2011 ; Felicitas SÁNCHEZ, 2012; Pamela ABELLÓN, 2012; Malena NIJENSOHN, 2012; María Inés LA GRECA, 2013; otros). Pasadas más de dos décadas luego de que fueran publicados las primeras traducciones y estudios filosóficos sobre Butler en Argentina, la aparición reciente de una serie de libros dedicados al estudio de su filosofía demuestra el interés que su obra continúa generando, así como también la apertura de nuevas y múltiples perspectivas interpretativas sobre su pensamiento. En la compilación titulada Judith Butler, su filosofía a debate (FEMENÍAS, Virginia CANO y TORRICELLA, 2013) es posible encontrar nuevas lecturas filosóficas acerca de las propuestas y "raíces conceptuales" de la obra de Butler. En otra compilación, Judith Butler. Las identidades del sujeto opaco (FEMENÍAS y Ariel MARTíNEZ, 2015), se rastrean las novedades en el pensamiento de Butler a la vez que se renueva el debate filosófico en torno a toda su obra. Dos lecturas sobre el pensamiento de Judith Butler (ABELLÓN y Magdalena DE SANTO, 2015) revisa el legado de Simone de Beauvoir en la obra de Butler y las tensiones entre las vertientes lingüística y dramática de la performatividad. Por su parte, Cuerpos en escena. Materialidad y cuerpo sexuado en Judith Butler y Paul B. Preciado (Martín DE MAURO RUCOVSKY, 2015) tensiona y hace dialogar a la teoría de la performatividad de Butler con la teoría de las incorporaciones prostéticas de Preciado.

También es posible encontrar numerosos trabajos que, en clave interdisciplinaria, revisan aspectos conceptuales de la obra de Butler que son relevantes para reflexionar acerca de los cuerpos, la política y la subjetividad en tanto producciones sociales e históricas (Andrea TORRICELLA, 2009; Eduardo MATTIO, 2010; Mariela PELLER, 2011 ; Nayla VACAREZZA, 2011; Mabel CAMPAGNOLI, 2013; SOLANA, 2014; Facundo Nazareno SAXE, 2015; Oriana SECCIA, 2015; Natalia SUNIGA, 2015; otros). Hallamos asimismo una serie de trabajos que comienzan a desarrollar modos productivos de utilización de los conceptos de Butler para construir objetos de estudio en el campo de los Estudios de Género y Sexualidades. En ellos, se analizan distintos problemas sociales locales tales como la solidaridad y la articulación del movimiento LGBT (MATTIO, 2009); la vida cotidiana y la militancia en las décadas del sesenta y setenta (Alejandra OBERTI, 2011); la seducción femenina (María Celeste BIANCIOTTI, 2011, 2012), la memoria queer (SAXE, 2012), la pornografía (SOLANA, 2013), la Ley de Identidad de Género (Paula AGUILAR y FERNÁNDEZ CORDERO, 2013) y la producción social de feminidades (VACAREZZA, 2015), entre otros. En este marco, se destacan los trabajos de Leticia Sabsay por haber aportado tanto agudas revisiones conceptuales de la obra de Butler (SABSAY, 2007, 2007a, 2008, 2009a, 2010; SOLEY-BELTRÁN y SABSAY, 2012), como también estudios de problemas sociales que se construyen y analizan utilizando las herramientas conceptuales de la teoría de la performatividad (SABSAY, 2009, 201 1, 2011 a, otros).

Para una mejor comprensión de la recepción crítica de Butler en Argentina, se postularon al comienzo del artículo cuatro nudos en torno a los cuales giró la polémica: 1) el uso de teorías provenientes de otras latitudes, 2) el estatuto de los cuerpos, 3) la crítica del capitalismo y 4) la relación de los conceptos con el activismo y la producción de conocimientos transformadores. Consideramos que dichos ejes permanecen abiertos en tanto preocupaciones recurrentes en la obra de Judith Butler, que se han ido desplegando de distintas maneras. Pero también, dichos nudos polémicos pueden ser considerados en 
tanto desafíos que pueden impulsar a mantener una perspectiva crítica acerca de los usos de la teoría de la performatividad.

En primer lugar y con respecto al uso de teorías provenientes de otras latitudes, es necesario recordar que, como argumenta Edward Said (1984), la vida intelectual se nutre y sostiene por la circulación de teorías que "viajan" a través de tiempos, situaciones y lugares. Para Butler, el uso productivo de las teorías en viaje exige un trabajo de "traducción cultural" que ella misma realiza en su obra donde convergen la llamada "teoría francesa" y las teorías de género estadounidenses (BUTLER, [1990] 2007). La traducción es, desde su punto de vista, siempre y cada vez un trabajo incierto en el cual el desafío es no "incorporar lo ajeno a un léxico establecido" (BUTLER, [2004] 2006, p. 64), sino transformar los lenguajes para que puedan aprehender lo otro en una relación mutua de desposesión productiva. Los conceptos de Judith Butler han viajado extensamente y su traducción ha sido amplia, al punto de que la intersección entre género, sexualidad y performatividad es hoy un espacio de interlocución trans-local que excede ese nombre de autora. Reflexionar críticamente sobre esas múltiples traducciones como ha señalado Leticia Sabsay (2014) implica pensar qué posibilidades tenemos de que nuestros usos, lecturas y producciones de conocimiento basados en esos conceptos lleven a su transformación y desestabilización. Es decir, el desafío es lograr ir más allá de la adaptación de teorías de otras latitudes a "contextos locales" para producir "casos". En un contexto global que, como sostiene Nelly Richard, "reserva el privilegio de la teoría a la academia metropolitana mientras la periferia latinoamericana descrita y analizada por esa teoría es vista como un simple campo de práctica" (RICHARD, 1996, p. 737-738), es fundamental insistir en la necesidad de pensar teóricamente y de entablar diálogos transnacionales que transformen los conceptos y la división del trabajo intelectual establecida entre centro y periferia.

En segundo lugar y en relación con el cuerpo, digamos que este tiene una importancia central a lo largo de la obra de Butler y no solo en la inicial reformulación del género como una práctica social performativa que se realiza sostenidamente por medio de actos corporales ritualizados (BUTLER, [1990] 1998; [1990] 2007). El cuerpo también es un eje central de su posterior crítica a la noción naturalista de "sexo" en la que planteó que ni este ni los cuerpos pueden considerarse como realidades ajenas a procesos de construcción cultural-discursiva (BUTLER, [1993] 2002). También, en sus más recientes elaboraciones acerca de la vulnerabilidad y la precariedad de los sujetos humanos, estos son considerados en tanto seres corpóreos (BUTLER, [2004] 2006a, [2005] 2009, [2009] 2010). Su trabajo ha sido reiteradamente acusado de convertir todo registro del cuerpo en discurso pero, sin embargo, esas lecturas pasan por alto las poderosas herramientas que la teoría de la performatividad provee para llevar adelante reflexiones y análisis que deconstruyan dicha dicotomía. La figura del quiasmo que Butler utiliza para dar cuenta de la relación entre cuerpo y lenguaje permite pensar ambos dominios como una trama cuyos hilos son difícilmente separables (VACAREZZA, 2011). En ese sentido, el desafío que propone esta perspectiva es no separar ni jerarquizar sino, por el contrario, mantener la tensión y señalar cómo procede la producción de cuerpos a través del lenguaje y, a la vez, dar cuenta del modo en que los cuerpos son un referente imposible, que provoca fallas, interrumpe y evade el lenguaje.

En tercer lugar y en relación con la crítica del capitalismo, Butler ha intervenido reiteradamente en debates de la izquierda radical y no ortodoxa (BUTLER, 1999a; BUTLER, Ernesto LACLAU y Slavoj ZIZEK, [2000] (2003). Los planteos de Butler en este sentido aportan a una comprensión renovada de los modos en los cuales las jerarquías de género y sexualidad se articulan con procesos de marginación y explotación propias del capitalismo global. En la obra de Butler es posible rastrear un esfuerzo sostenido por comprender los procesos por los cuales distintas jerarquías (de clase, de raza, sexuales, etc.) se articulan de formas 
complejas sin privilegiar ni reificar esas diferencias como características inherentes a sujetos individuales o colectivos. Las reflexiones y análisis recientes de Butler sobre la vulnerabilidad, la precariedad y la desposesión avanzan en esa dirección (2006, 2006a, 2009, 2010; BUTLER y Athena ATHANASIOU, 2013; BUTLER, 2015), focalizando sobre la dimensión colectiva de la performatividad y sobre la resistencia como un acto corporal. En su elaboración última de una ontología social centrada en la relacionalidad y la interdependencia humana, muestra los modos en que formas extremas de vulnerabilidad y precariedad son inducidas sistemáticamente sobre determinadas poblaciones por medios económicos que, en el contexto del dominio totalitario del capitalismo, no dejan de intersectarse con lógicas de dominación racista, colonialista y heterosexista.

En cuarto lugar, mencionamos el desafío que supone no desconocer la compleja y disputada relación de ciertos usos de la teoría de la performatividad con los activismos y la producción de conocimientos. En cuanto a las cuestiones trans, resulta necesario en este punto recordar que, en Argentina, las conceptualizaciones de Butler fueron tempranamente apropiadas tanto por los feminismos como por los emergentes activismos queer y trans. En esa coyuntura, los textos de Butler fueron utilizados como herramientas en la construcción de saberes críticos que, dentro y fuera de la academia, buscaron impulsar las demandas de las comunidades trans. Sin embargo, no todos los usos han resultado políticamente productivos $y$, de hecho, algunos han generado intensas polémicas. Los deslizamientos hacia formas de objetualización y exotización realizadas en nombre del quehacer académico han encontrado una fuerte resistencia por parte de travestis y trans que han criticado públicamente este tipo de abordajes (cualquiera sean las herramientas teóricas esgrimidas) (FIGARI, 2014a). Butler misma ha sido interpelada sobre estas cuestiones (RADI, 2015a), que permanecen abiertas a la polémica. La resistencia a la objetualización está ligada a una firme y valiosa apuesta política del activismo trans por habitar la academia, construir autoridad epistémica, conocimientos y formas de teorización propias. A un nivel más general, el desafío que se plantea, entonces, al intentar articular conceptos con el activismo y la producción de conocimiento crítico, no radica en proclamar la validez o la ineficacia de la teoría de la performatividad de Butler o de otras herramientas conceptuales para el estudio de tal o cual problema social, sino de reflexionar acerca de qué tipo de traducciones, apropiaciones creativas y alianzas ético-políticas deben articularse para la producción de saberes críticos que puedan intervenir en la transformación social.

Trazamos hasta aquí un mapa incompleto de las derivas, debates y desafíos que plantea el uso de la teoría de la performatividad en Argentina. Sin dudas, este mapa debe permanecer abierto debido al carácter proliferante del territorio que intentamos cartografiar. Su amplitud puede comprenderse en los términos de cierto establecimiento de los conceptos de la teoría de la performatividad como puntos de referencia ineludibles a la hora de reflexionar acerca del género, los cuerpos y las sexualidades. Pero, además de la reiteración de la cita obligada por las normas del campo disciplinar, es posible advertir también, en esas repeticiones, la intensidad de un diálogo, la hondura de las polémicas y la productividad de conceptos que, en el uso, van transformándose y excediendo, sin dudas, lo dicho por su autora.

Nos encontramos, en Argentina, con una recepción de los conceptos de Judith Butler que, desde sus inicios, no puede caracterizarse como cerrada en un ámbito académico desprovisto de conexiones con las luchas sociales. En muchos casos se trata de trabajos realizados por activistas y personas interesadas en intervenir y colaborar con sus investigaciones en disputas políticas presentes. En ese tránsito es posible advertir la productividad y fuerza performativa de una teoría, que sigue inquietando, generando discusiones y siendo traducida de un territorio a otro. 


\section{Agradecimiento}

Este trabajo es una versión ampliada del estudio realizado para elaborar el estado de la cuestión mi tesis doctoral. Agradezco a July Chaneton, Laura Fernández Cordero, Fernanda Carvajal, Joaquín Insausti y Barbara Sutton los diálogos mantenidos durante la elaboración de este artículo que pude completar gracias a la generosa invitación de esta última para realizar una estancia académica en la University at Albany.

\section{Referencias}

ABELLÓN, Pamela. Espectros beauvorianos en la obra de Judith Butler. 2012. Tesis de Licenciatura en Filosofía? Universidad de Buenos Aires, Buenos Aires.

ABELLÓN, Pamela y DE SANTO, Magdalena. Dos lecturas sobre el pensamiento de Judith Butler. Villa María: Eduvim, 2015.

ACHA, Omar; BEN, Pablo; D'ANTONIO, Débora; HALPERÍN, Paula y LAGO, Daniela. "Políticas identitarias y políticas socialistas". El rodaballo, n. 11/12, p. 92-100, primavera, 2000.

ACZEL, Ilona y PÉCHIN, Juan. "Las reformas del Código Contravencional de la Ciudad Autónoma de Buenos Aires: Regulaciones democráticas y represión política". En: ÁREA QUEER DE LA FACULTAD DE FILOSOFÍA Y LETRAS, UBA. Código Contravencional de la Ciudad de Buenos Aires. Documentos, normas, debates y luchas políticas. Buenos Aires: Formato CD ROM, 2006.

AGUILAR, Paula y FERNÁNDEZ CORDERO, Laura. "Cuando la identidad es ley. Ecos de Michel Foucault y Judith Butler”. En: TORRES, Mónica; SCHNITZER, Graciela; ANTUÑA, Alejandra y PEIDRO, Santiago (comps.). TRANSformaciones. Ley, diversidad, sexuación. Buenos Aires: Grama, 2013. p. 45-55.

ALMA, Amanda y LORENZO, Paula. Mujeres que se encuentran. Una recuperación histórica de los Encuentros Nacionales de Mujeres en Argentina (1986-2005). Buenos Aires: Feminaria, 2009.

AMADO, Ana. "Cuerpos intransitivos. Los debates feministas sobre la identidad". Debate feminista, año 11 , v. 21, p. 233-240, abril, 2000.

ÁREA QUEER DE LA FACULTAD DE FILOSOFÍA Y LETRAS, UBA. Código Contravencional de la Ciudad de Buenos Aires. Documentos, normas, debates y luchas políticas. Buenos Aires: Formato CD ROM, 2006.

AZARAF, Liliana; CATALÁ, Silvia; DAUNES, Liliana; D'UVA, Mónica; FERNÁNDEZ, Josefina y VICENTE, Silvia. "Las desventuras del género". Feminaria, n. 24/25, p. 54-56, noviembre, 2000.

AZCÁRATE, Teresa; BARTíS María Elena y WERTHEIN, Silvia. "Tensiones feministas: Resignificando lo políico". Anuario de Hojas de Warmi, p. 103-113, 1999.

BACCl, Claudia; FERNÁNDEZ CORDERO, Laura y OBERTI, Alejandra. "De injusticias y anacronismos". El rodaballo, n.11/12, p. 86-91, primavera, 2000.

BELLUCCI, Mabel. "Las primeras lecturas". Suplemento Las 12, Diario Página/12, 25 de septiembre de 2015.

BELLUCCI, Mabel y RAPISARDI, Flavio. "Alrededor de la identidad: Las luchas políticas del presente". Nueva sociedad, n. 162, p. 40-53, julio/agosto, 1999.

BERKINS, Lohana. "Eternamente atrapadas por el sexo". En: FERNÁNDEZ, Josefina, D'UVA, Mónica y VITURRO, Paula (comps.). Cuerpos ineludibles: Un diálogo a partir de las sexualidades en América Latina. Buenos Aires: Ají de Pollo, 2004. p. 19-24.

BERKINS, Lohana (comp.). Cumbia, copeteo y lágrimas. Informe nacional sobre la situación de las travestis, transexuales y transgéneros. Buenos Aires: Asociación de Lucha por la Identidad Travesti-Transexual, 2007. 
BERKINS, Lohana. "Un itinerario político del travestismo". En: MAFFÍA, Diana (comp.). Sexualidades migrantes. Género y transgénero. Buenos Aires: Librería de Mujeres y Feminaria, 2008. p. 145-155.

BERKINS, Lohana. "Travestismo, transexualidad y transgeneridad". En: RAICES MONTERO, Jorge Horacio (comp.). Un cuerpo: Mil sexos. Intersexualidades. Buenos Aires: Topia, 2010. p. 91-102.

BERKINS, Lohana. "Los existenciarios trans". En: FERNÁNDEZ, Ana María y SIQUEIRA PERES, William (comps.). La diferencia desquiciada. Géneros y diversidades sexuales. Buenos Aires: Biblos, 2013. p. 91-96.

BERKINS, Lohana y FERNÁNDEZ, Josefina (coords.). La gesta del nombre propio. Informe sobre la situación de la comunidad travesti en la Argentina. Buenos Aires: Ediciones Madres de Plaza de Mayo, 2005.

BERKINS, Lohana y JONES, Daniel. "Entrevista con Lohana Berkins". 2008. Disponible en: http://www.clam.org.br/uploads/arquivo/Entrevista\%20con\%20Lohana\%20Berkins(1).pdf. Acceso en: 14 de octubre de 2016.

BIANCIOTTI, María Celeste. "Cuerpo y género: apuntes para pensar prácticas eróticas de mujeres jóvenes. Aportes de Judith Butler y Pierre Bourdieu". Revista Latinoamericana de Estudios sobre Cuerpos, Emociones y Sociedad, n. 6, p. 70-82, agosto/noviembre, 2011.

"Sobre performances y efectos performativos: género, juventud y seducción femenina". Revista Latinoamericana de Sexualidad, Salud y Sociedad, n. 8, p. 63-89, abril/julio, 2012.

BOURDIEU, Pierre. Intelectuales, política y poder. Buenos Aires: Eudeba, 1999.

BUTLER, Judith. "Problemas de los géneros, teoría feminista y discurso psicoanalítico". En: NICHOLSON, Linda (comp.). Feminismo/Posmodernismo. Buenos Aires: Feminaria Editora, 1992. p. 75-95.

BUTLER, Judith. "Sujetos de sexo/género/deseo". Feminaria, n. 19, p. 1-20, junio, 1997.

BUTLER, Judith. "Actos performativos y constitución del género: un ensayo sobre fenomenología y teoría feminista". Debate feminista, año 9, n. 18, p. 296-314, octubre, 1998.

BUTLER, Judith. "Sexo y género en El segundo sexo de Simone de Beauvoir". Mora, n. 4, p. 1021 , octubre, 1998a.

BUTLER, Judith. "El primer libro de Judith Butler: Los problemas de género". Mora, n. 4, p. 22 26, octubre, 1998b.

BUTLER, Judith. "La vida psíquica del poder. Teorías de la sujeción. Introducción". Feminaria, n. 22/23, p. 1-13, julio, 1999.

BUTLER, Judith. "Meramente cultural". El rodaballo, n. 9, p. 53-58, verano, 1999a.

BUTLER, Judith. "Imitación e insubordinación de género". En: AA.W. Grafías de Eros. Historia, género e identidades sexuales. Buenos Aires: Ediciones de la École Lacanniene de Psychanalyse, 2000. p. 87-113.

BUTLER, Judith. Cuerpos que importan. Sobre los límites materiales y discursivos del "sexo". Buenos Aires: Paidós, 2002.

BUTLER, Judith. Deshacer el género. Barcelona: Paidós, 2006.

BUTLER, Judith. Vida precaria. El poder del duelo y la violencia. Buenos Aires: Paidós, 2006a.

BUTLER, Judith. El género en disputa. El feminismo y la subversión de la identidad. Barcelona: Paidós, 2007.

BUTLER, Judith. Dar cuenta de sí mismo. Violencia ética y responsabilidad, Buenos Aires: Amorrortu, 2009.

BUTLER, Judith. Marcos de guerra. Las vidas Iloradas. Buenos Aires: Paidós, 2010. 
BUTLER, Judith. Notes Toward a Performative Theory of Assembly. Cambridge: Harvard University Press, 2015.

BUTLER, Judith y ATHANASIOU, Athena. Dispossession: The Performative in the Political. Cambridge: Polity Press, 2013.

BUTLER, Judith; LACLAU, Ernesto y ZIZEK, Slavoj. Contingencia, hegemonía y universalidad. Diálogos contemporáneos en la izquierda. Buenos Aires: Fondo de Cultura Económica, 2003.

BUTLER, Judith y SCOTT, Joan. "Las feministas teorizan lo político". Feminaria, n. 20, p. 15-17, octubre, 1997.

CABRAL, Mauro. "Pensar la intersexualidad, hoy". En: MAFFÍA, Diana (comp.). Sexualidades migrantes. Género y transgénero. Buenos Aires: Librería de Mujeres y Feminaria, 2008. p. 131-142.

CABRAL, Mauro. (comp.). Interdicciones. Escrituras de la transexualidad en castellano. Córdoba: Anarrés, 2009.

CABRAL, Mauro. "El doble acceso a la identidad". 2011 . Disponible en: http://www.clam.org.br/ publique/cgi/cgilua.exe/sys/start.htm?infoid=9064\&sid=25. Acceso en: 14 de octubre de 2016.

CABRAL, Mauro. "Algo ha pasado". En: MORÁN FAÚNDES, José Manuel; SGRO RUATA, María Candelaria y VAGGIONE, Juan Marco. Sexualidades, desigualdades y derechos. Reflexiones en torno a los derechos sexuales y reproductivos. Córdoba: Ciencia, Derecho y Sociedad, 2012. p. 251-273.

CABRAL, Mauro y VITURRO, Paula. "(Trans)Sexual Citizenship in Contemporary Argentina". En: CURRAH, Paisley; JUANG, Richard y MINTER, Shannon (eds.). Transgender Rights. Minneapolis: University of Minnesota Press, 2006. p. 262-273.

CAMPAGNOLI, Mabel. "La noción de quiasmo en Judith Butler: para una biopolítica positiva". Nómadas, n. 39, p. 47-61, octubre, 2013.

CASALE, Rolando. "Deseo y producción de agencia en Judith Butler". En: FEMENÍAS, María Luisa (comp.). Feminismos de París a La Plata. Buenos Aires: Catálogos, 2006. p. 67-80.

CASALE, Rolando y CHIACCHIO, Cecilia (comps.). Máscaras del deseo. Una lectura del deseo en Judith Butler. Buenos Aires: Catálogos, 2009.

CHÁNETON, July. "La ciudad de las damas. Teorías y prácticas feministas". Nueva sociedad, n. 155, p. 37-53, mayo/junio, 1998.

CIRIZA, Alejandra. "Notas sobre los límites de la importación teórica. A propósito de Judith Butler". El rodaballo, n. 15, p. 58-60, invierno, 2004.

CIRIZA, Alejandra. "A propósito de la pregunta por el sujeto en algunos escritos de Judith Butler. Sobre las relaciones entre filosofía y psicoanálisis". En: DALMASSO, María Teresa y BORIA, Adriana (eds.). Discurso social y construcción de identidades. Mujer y género. Córdoba: Centro de Estudios Avanzados - Universidad Nacional de Córdoba, 2006. p. 31-44.

CIRIZA, Alejandra. "A propósito de una controversia feminista: sobre ambivalencias conceptuales y asuntos de disputa. Las relaciones entre cuerpo y política". Herramienta, n. 45, octubre, 2010. Disponible en: http://www.herramienta.com.ar/revista-herramienta$\mathrm{n}$-45/proposito-de-una-controversia-feminista-sobre-ambivalencias-conceptuales-y-. Acceso en: 14 de octubre de 2016.

D'ATRI, Andrea. "Feminismo y democracia en Judith Butler. Entre la metonimia del mercado y la metáfora (imposible) de la revolución". Estrategia internacional, n. 20, p. 215-224, 2003. Disponible en: http://www.ft.org.ar/estrategia/ei20/ei20feminismo.htm. Acceso en: 14 de octubre de 2016. 
DELFINO, Silvia. "Genero y regulaciones culturales. El valor crítico de las diferencias". En: FORASTELLI, Fabricio y TRIQUELL, Ximena (comps.). Las marcas del género: configuraciones de la diferencia en la cultura. Córdoba: Centro de Estudios Avanzados - Universidad Nacional de Córdoba, 1999. p. 67-84.

DELFINO, Silvia y RAPISARDI, Flavio. "Cuirizando la cultura argentina desde La Queerencia. Centro criollo de políticas de la diferencia". Ramón, n. 99, p. 10-14, abril, 2010.

DELFINO, Silvia y SALOMÓN, Guadalupe. "Regulaciones culturales y luchas políticas: El caso del Código Contravencional de la Ciudad de Buenos Aires". Revista Jurídica Universidad Interamericana de Puerto Rico, v. 38, n. 1, p. 151-168, septiembre/diciembre, 2003.

DE MAURO RUCOVSKY, Martín. Cuerpos en escena. Materialidad y cuerpo sexuado en Judith Butler y Beatriz Preciado. Barcelona: Egales, 2015.

DOMÍNGUEZ, Nora; CASTRO, Marcela; CHÁNETON, July; DASZUK, Silvana y JUROVIETZKY, Silvia. "Sujeto, género y discurso: ¿Sos o te hacés?". Hiparquia, v. VII, noviembre, 1994. Disponible en: http://www.hiparquia.fahce.unlp.edu.ar/numeros/volvii/sujeto-genero-y-discursobfsos-o-te-haces. Acceso en: 14 de octubre de 2016.

ESPINOSA MIÑOSO, Yuderkis. "A una década de la performatividad: de presunciones erróneas y malos entendidos". En: FERNÁNDEZ, Josefina; D'UVA, Mónica y VITURRO, Paula (comps.). Cuerpos ineludibles: Un diálogo a partir de las sexualidades en América Latina. Buenos Aires: Ají de Pollo, 2004. p. 143-162.

FEMENÍAS, María Luisa. "Butler lee a Beauvoir: Fragmentos de una polémica en torno del "sujeto»". Mora, n. 4, p. 4-9, octubre, 1998.

FEMENÍAS, María Luisa. "Butler y Beauvoir en diálogo imposible". Travesías, n. 8, p. 43-53, 1999.

FEMENÍAS, María Luisa. Judith Butler: Introducción a su lectura. Buenos Aires: Catálogos, 2003.

FEMENÍAS, María Luisa. "El feminismo académico en Argentina". Labrys. Estudos feministas/ Études féministes, n. 7, enero/julio, 2005. Disponible en: http://www.labrys.net.br/labrys7/ fem/mluisa.htm. Acceso en: 14 de octubre de 2016.

FEMENÍAS, María Luisa. Sobre sujeto y género: (Re)Lecturas feministas desde Beauvoir a Butler. Buenos Aires: Prohistoria, 2012.

FEMENÍAS, María Luisa. "Butler, la muerte del Hombre y el sujeto opaco". Revista de psicoanálisis, tomo LXX, n. 2/3, 2013. p. 349-370.

FEMENÍAS, María Luisa; CANO, Virginia y TORRICELLA, Paula (comps.). Judith Butler, su filosofía a debate. Buenos Aires: Editorial de la Facultad de Filosofía y Letras de la Universidad de Buenos Aires, 2013.

FEMENÍAS, María Luisa y MARTíNEZ, Ariel. Judith Butler: Las identidades del sujeto opaco. La Plata: Universidad de La Plata, 2015.

FERNÁNDEZ, Josefina. Cuerpos desobedientes. Travestismo e identidad de género. Buenos Aires: Edhasa, 2004.

— "El género en llamas. Travestismo e identidades genéricas". Feminaria, n. 30/31, p. 2535, abril, 2007.

FERNÁNDEZ, Josefina, D'UVA, Mónica y VITURRO, Paula (comps.). Cuerpos ineludibles: Un diálogo a partir de las sexualidades en América Latina. Buenos Aires: Ají de Pollo, 2004.

FIGARI, Carlos. "Queer Argie". American Querterly, v. 66, n. 3, p. 621-631, septiembre, 2014.

— "Fagocitando lo queer en el Cono Sur". En: FALCONÍ TRÁVEZ, Diego; CASTELLANOS, Santiago y VITERI, María Amelia (eds.). Resentir lo queer en América Latina. Diálogos desde/con el Sur. Barcelona: Egales, 2014a. p. 63-79. 
FORASTELLI, Fabricio. "Regulaciones culturales y violencia. Recientes debates en el movimiento de mujeres y queer en América Latina". Feminismo/s, n. 9, p. 51-66, junio, 2007.

FORASTELLI, Fabricio. "Los debates sobre el género y lo queer y luchas políticas en Argentina". En: COMINS MINGOL, Irene y PARÍS ALBERT, Sonia (comps.). Investigación para la paz: Estudios filosóficos. Barcelona: Icaria, 2010. p. 157-170.

FRASER, Nancy. "Heterosexismo, no-reconocimiento y capitalismo: Una respuesta a Judith Butler". El rodaballo, n. 10, p. 25-28, verano, 2000.

HALPERÍN, Paula y ACHA, omar. Cuerpos, géneros e identidades. Estudios de historia de género en Argentina. Buenos Aires: Ediciones del Signo, 2000.

HEINÄMAA, Sara. "¿Qué es ser mujer? Butler y Beauvoir sobre los fundamentos de la diferencia sexual". Mora, n. 4, p. 27-44, octubre, 1998.

LA GRECA, María Inés. Historia, figuración y performatividad: Crítica y persistencia de la narración en la Nueva Filosofía de la Historia. 2013. Tesis de Doctorado en Filosofía? Universidad de Buenos Aires, Buenos Aires.

MAFFÍA, Diana (comp.). Sexualidades migrantes. Género y transgénero. Buenos Aires: Librería de Mujeres y Feminaria, 2008.

MARISTANY, José. "Del pudor en el lenguaje: notas sobre lo queer en Argentina". Lectures du genre, n. 10, p. 102-111, 2013.

MATTIO, Eduardo. "Todos somos vulnerables. Algunos aportes para la construcción de una solidaridad LGTB en Judith Butler". JORNADAS CINIG DE ESTUDIOS DE GÉNERO Y FEMINISMOS, I, Universidad Nacional de La Plata, 2009.

MATTIO, Eduardo. "Vulnerabilidad, normas de género y violencia estatal: ontología social y política sexual en la última Judith Butler". Pensamento plural, n. 7, p. 159-172, julio/ diciembre, 2010.

NIJENSOHN, Malena. Escrituras de la subjetividad: de Nietzsche a Butler. 2012. Tesis de Licenciatura en Filosofía - Universidad de Buenos Aires, Buenos Aires.

OBERTI, Alejandra. Género, política y violencia: vida cotidiana y militancia en las décadas del sesenta y setenta. 2011. Tesis de Doctorado en Ciencias Sociales - Universidad de Buenos Aires, Buenos Aires.

PALMEIRO, Cecilia. Desbunde y felicidad. De la cartonera a Perlongher. Buenos Aires: Título, 2012.

PALMEIRO, Cecilia. "Derivas de lo queer en la Argentina: hacia una genealogía". Periódicus, v. 1, n. 1, p. 21-42, mayo/octubre, 2014.

PELLER, Mariela. "Judith Butler y Ernesto Laclau: debates sobre la subjetividad, el psicoanálisis y la política". Sexualidad, Salud y Sociedad, n. 7, p. 44-68, abril, 2011.

RADI, Blas. "Algunas consideraciones sobre 'el binario' y la Ley de Identidad de Género en Argentina". 2013. Disponible en: http://www.aacademica.org/blas.radi/8.pdf. Acceso en: 14 de octubre de 2016.

RADI, Blas. "Serás activista trans o... serás activista trans: sobre el consumo responsable de información”. La revista del CCC, n. 22, p. 1-6, enero/junio, 2015.

RADI, Blas. "Economía del privilegio". Suplemento Las 12, Diario Página/12, 25 de septiembre de 2015a.

RAPISARDI, Flavio. "Identidad política y diferencia: El problema de la constitución de una identidad homosexual en los "gay and lesbian studies" y la "queer theory". Doxa. Cuadernos de Ciencias Sociales, año VII, n. 17, p. 9-13, otoño, 1997.

RAPISARDI, Flavio. "Crítica y diferencia: Sobre las políticas queer de emancipación". La Gandhi Argentina, año II, n. 3, p. 32-33, noviembre, 1998. 
RAPISARDI, Flavio. "Estudios Culturales: Una cartografía". El grito, año I, n. 1, p. 37-38, enero, 1999.

RAPISARDI, Flavio. "Sobre los límites de la ciudadanía liberal: identidades, clase y trabajo en la lucha contra el Código Contravencional Porteño". Mora, n. 11, p. 167-189, diciembre, 2005.

RAPISARDI, Flavio. "Escritura y lucha política en la cultura argentina: Identidades y hegemonía en el movimiento de diversidades sexuales entre 1970 y 2000 ". Revista Iberoamericana, v. LXXIV, n. 225, p. 973-995, octubre/diciembre, 2008.

RICHARD, Nelly. "Feminismo, experiencia y representación”. Revista Iberoamericana, v. LXII, n. 176/177, p. 733-744, julio/diciembre, 1996.

SABSAY, Leticia. Los dilemas del antiesencialismo en la teoría feminista contemporánea: una reflexión en torno a Judith Butler. Almería: Instituto de Estudios Almerienses, 2007.

SABSAY, Leticia. "La voz del cuerpo entre la materialidad y la significación". En: AA.W. Oberturas del cos / Aberturas del Cuerpo. Valencia: Servicio de Publicaciones de la Universidad de Valencia, 2007a. p. 43-48.

SABSAY, Leticia. "Deseo y discurso en el sujeto (feminista) de la performatividad". En: ARFUCH, Leonor y CATANZARO, Gisela (comps.). Pretérito imperfecto. Lecturas críticas del acontecer. Buenos Aires: Prometeo, 2008. p. 187-217.

SABSAY, Leticia. Las normas del deseo. Imaginario sexual y comunicación. Madrid: Cátedra, 2009.

SABSAY, Leticia. "Tras la firma de Judith Butler. Una introducción posible a los ecos de su escritura". Revista de Antropología Iberoamericana, v. 4, n. 3, p. 311 -320, septiembre/ diciembre, 2009a.

SABSAY, Leticia. "¿En los umbrales del género? Beauvoir, Butler y el feminismo ilustrado". Feminismo/s, n. 15, p. 119-135, junio, 2010.

SABSAY, Leticia. Fronteras sexuales. Espacio urbano, cuerpos y ciudadanía. Buenos Aires: Paidós, 2011.

SABSAY, Leticia. "The Limits of Democracy. Transgender Work and Citizenship". Cultural Studies, v. 25, n. 2, p. 213-229, marzo, 2011 a.

SABSAY, Leticia. "Políticas queer, ciudadanías sexuales y decolonización". En: FALCONÍ TRÁVEZ, Diego; CASTELLANOS, Santiago y VITERI, María Amelia (eds.). Resentir lo queer en América Latina. Diálogos desde/con el Sur. Barcelona: Egales, 2014. p. 45-58.

SAID, Edward. "Traveling Theory". En: SAID, Edward. The World, the Text and the Critic. Cambridge: Harvard University Press, 1984. p. 226-247.

SALOMÓN, Guadalupe. "Luchas identitarias de sujetos y colectivos travestis, transexuales y transgéneros. La práctica discursiva del derecho como configurador de identidades de género". En: COLOQUIO DE INVESTIGACIÓN: GÉNERO Y CIENCIAS SOCIALES, I. BuenOS Aires: IIEGE, FFyL, UBA y Servicio Alemán de Intercambio Académico, 2011.

SALOMÓN, Guadalupe. "La materia contravencional sale del closet". En: COLOQUIO DE INVESTIGACIÓN: GÉNERO Y CIENCIAS SOCIALES, II. Buenos Aires: IIEGE, FFyL, UBA y Servicio Alemán de Intercambio Académico, 2012.

SÁNCHEZ, Felicitas. Judith Butler: ontología para un concepto de ciudadanía democrática radical. 2012. Tesis de Licenciatura en Filosofía, Universidad de Buenos Aires, Buenos Aires.

SAXE, Facundo Nazareno. "Dictaduras, narrativa y sexualidad disidente: un enfoque comparatístico de la literatura de la memoria queer". Cuadernos del sur - Letras, n. 42, p. 265-276, 2012.

SAXE, Facundo Nazareno. "La noción de performatividad en el pensamiento de Judith Butler: queerness, precariedad y sus proyecciones", Estudios Avanzados, n. 24, p. 1-14, diciembre, 2015. 
SCHWARZ, Roberto. "Las ideas fuera de lugar". Meridional. Revista chilena de estudios latinoamericanos, n. 3, p. 183-199, octubre, 2014.

SECCIA, Oriana. "Entre los afectos y los discursos: la producción identitaria en Judith Butler". Entramados y perspectivas, v. 5, n. 5, p.153-177, septiembre, 2015.

SOLANA, Mariela. La noción de subversión en Judith Butler: reflexiones sobre su formación, sus críticas y su aplicabilidad. 2011 . Tesis de Licenciatura en Filosofía - Universidad de Buenos Aires, Buenos Aires.

SOLANA, Mariela. "Pornografía y subversión: una aproximación desde la teoría de género de Judith Butler". Convergencia. Revista de Ciencias Sociales, v. 20, n. 62, p. 159-179, mayo/agosto, 2013.

SOLANA, Mariela. "El papel del travestismo en el pensamiento político de Judith Butler". Revista de Filosofía y Teoría Política, n. 45, p. 1-26, diciembre, 2014.

SOLEY-BELTRÁN, Patricia y SABSAY, Leticia (eds.). Judith Butler en disputa. Lecturas sobre la performatividad. Barcelona: Egales, 2012.

SUNIGA, Natalia. "El falo en disputa. Judith Butler, lectora crítica de Jacques Lacan", Diferencias. Revista de teoría social contemporánea. v. 1, n. 1, p. 64-86, noviembre, 2015.

TARCUS, Horacio. Marx en la Argentina. Sus primeros lectores obreros, intelectuales y científicos. Buenos Aires: Siglo Veintiuno, 2007.

TARCUS, Horacio. El socialismo romántico en el Río de la Plata (1837-1852). Buenos Aires: Fondo de Cultura Económica, 2016.

TORRICELLA, Andrea. "La relación lenguaje-cuerpo-performatividad en la obra de Judith Butler: una cartografía”. Debate feminista, año 20, v. 40, p. 229-239, octubre, 2009.

TORRICELLA, Paula. "Reflexiones en torno a la recepción de una obra". En: FEMENÍAS, María Luisa; CANO, Virginia y TORRICELLA, Paula (comps.). Judith Butler. Su filosofía a debate. Buenos Aires: Editorial de la Facultad de Filosofía y Letras - UBA, 2013. p. 173-189.

VACAREZZA, Nayla. "Figuraciones del cuerpo con género. Paralelismo y quiasmo". Revista Latinoamericana de Estudios sobre Cuerpos, Emociones y Sociedad, n. 6, p. 33-43, agosto/noviembre, 2011.

VACAREZZA, Nayla. Feminidades en la encrucijada de los estilos corporales y las categorías identitarias de género y sexualidad. 2015. Tesis de Doctorado en Ciencias Sociales Universidad de Buenos Aires, Buenos Aires.

WAYAR, Marlene. "Carta a Nadia Echazú". En: FERNÁNDEZ, Josefina y BERKINS, Lohana (coords.). La gesta del nombre propio. Informe sobre la situación de la comunidad travesti en la Argentina. Buenos Aires: Ediciones Madres de Plaza de Mayo, 2005. p. 6769.

WAYAR, Marlene. "La visibilidad de lo invisible". En: BERKINS, Lohana (comp.). Cumbia, copeteo y lágrimas. Informe nacional sobre la situación de travestis, transexuales y transgéneros. Buenos Aires: Asociación de Lucha por la Identidad Travesti-Transexual, 2007. p. 43-54.

WAYAR, Marlene. “¿Qué pasó con la T?”. Suplemento Soy, Página/12, 11 de mayo de 2012. WAYAR, Marlene. "No quiero invisibilizarme detrás de una mujer". En: TORRES, Mónica; SCHNITZER, Graciela; ANTUÑA, Alejandra y PEIDRO, Santiago (comps.). TRANSformaciones. Ley, diversidad, sexuación. Buenos Aires: Grama, 2013. p. 69-84.

[Recebido em 07/11/2015, reapresentado em $24 / 10 / 2016$ e aprovado em 13/02/2017] 


\begin{abstract}
Judith Butler in Argentina. Reception and polemic around the theory of gender performativity

Abstract: In Spanish language, the ideas and concepts of Judith Butler have circulated for more than two decades. Its reception was not uniform since, in every Spanish-speaking country, it was conditioned by the interest and the resistance that her daring propositions produced on feminisms and Gender Studies, by the anachronisms of translation and by the dynamics of the academy and the publishing industry. In Argentina, the first translations of Butler's texts began to be published and circulated in the nineties, in magazines that were neither fully devoted to theory or academic work, nor devoted to activism devoid of academic concepts and ways of thinking. This article explores the different forms of reception, reading and use of the concepts of gender performativity theory in Argentina, as well as the controversies that were generated around it focusing on the tensions and intersections between activism and academy. These fields can be thought of as spaces of disputes with much more fragile and porous boundaries than often assumed.

Key words: Judith Butler; performativity; reception; Argentina
\end{abstract}

Nayla Luz Vacarezza (nayla.luz@gmail.com ): Licenciada y profesora en Sociología por la Universidad de Buenos Aires, donde también obtuvo el título de doctora en Ciencias Sociales. Se desempeña como auxiliar docente en la carrera de Sociología. Fue becaria doctoral del Consejo Nacional de Investigaciones Científicas y Técnicas de Argentina y actualmente es becaria postdoctoral de la misma institución. Es investigadora del proyecto UBACYT "La experiencia social de los límites. Cuerpos, hablas, poderes" y forma parte del Grupo de Trabajo "Micropolíticas visuales de los afectos" (Universidad Nacional de La Plata). 